\title{
Effect of Monopole Field on the Non-spherical Gravitational Collapse of Strange-quark Matter.
}

\author{
S. S. Zade ${ }^{1}$, C. S. Khodre ${ }^{2 *}$ and K. D. Patil ${ }^{3}$ \\ ${ }^{I}$ Department of Mathematics,J.B. College of Science, Wardha (M.S.), India \\ ${ }^{2}$ Department of Mathematics,S.D.College of Engineering, Selukate, Wardha (M.S.), India \\ ${ }^{3}$ Department of Mathematics, B.D.College of Engineering, Sevagram, Wardha (M.S.), India
}

\begin{abstract}
We investigate the nature of the singularity in the non-spherical gravitational collapse of the strange quark null fluid. The interesting feature that emerges is that the non-spherical gravitational collapse of charged strange quark matter leads to a naked singularity whereas the gravitational collapse of neutral monopole quark matter gives the singularity is covered in an event horizon, i.e. it produces a black hole. In the present paper we have shown that the monopole spacetime does not play any fundamental roll in the formation of naked singularity.
\end{abstract}

Keywords: Cosmic censorship, event horizon, gravitational collapse, naked singularity.

\section{Introduction}

In the past fourty years, there have been extensive studies on gravitational collapse investigating thenature of singularities. The study of gravitational collapse of spherically symmetric spacetimes has led to many examples of naked singularities.[1-10] Most of the studies concentrated on the aim to find light rays emanating from the central singularity and escaping the Schwarzschildian trapped surface at least locally. In order to study the nature of the singularity, global behaviour of radial null geodesics must be studied in full generality.

The cosmic censorship conjecture (CCC) articulated by Penrose [11] is fundamental to many aspects of theory and astrophysical applications of black hole physics today. Despite many attempts over past decades no theoretical proof or even any satisfactory mathematical formulation of CCC is available as of today in the case of dynamical gravitational collapse. In the mean time, many authors have studied mainly spherical gravitational collapse of a massive matter cloud within the framework of general relativity. As the nuclear fuel of a massive star exhausts, it loses its equilibrium and gravity becomes the central dominant force which lends the star to its perpetual collapse. The gravitational collapse studies then show that the collapse end state is either a black hole (BH) or a naked singularity (NS), depending on the nature of the initial data from which the collapse evolves, arising from a regular initial state to the final super dense state [12-18]. The works such as above analyze physically relevantgeneral matter fields that include most of the known physical forms of matter like dust, perfect fluids, and such others. Wide classes of solutions to Einstein equations are shown in these cases to exist where the collapse end state is a black hole or naked singularity depending on the nature of initial data and the collapse evolutions allowed by the Einstein equations subject to regularity and physical reasonability conditions. One of the hot topics in astrophysics of late has been the possibility of the discovery of strange stars. A quark star or strange star is a hypothetical type of star composed of quark matter or strangematter. It is theorized that when the neutron degenerate matter which makes up a neutron star is put under sufficient pressure due to the star's gravity, the individual neutrons break down into their constituent quarks - up quarks and down quarks. Some of these quarks may then become strange quarks and form strange matter. The star which then becomes is known as a quark star or strange star. The broader meaning of strange matter is just quark matter that contains three flavours of quarks up, down and strange. In this definition, there is a critical pressure and an associated critical density; and when nuclear matter is compressed beyond this density, the protons and neutrons dissociate into quarks, yielding strange matter. Many research papers on the strange quark matter have appeared so far, explaining the formation and properties of strange stars [19-23]. The strange quark matter is characterized by the equation of state

$$
P=\frac{\rho-4 B}{3}
$$

where $B$ is the difference between the energy density of the perturbative and nonperturbativeQCD vacuum, known as the bag constant. $\rho$ and $P$ are respectively, the energy density and thermodynamic pressure of the quark matter. The typical value of the bag constant is of the order of $1015 \mathrm{~g} / \mathrm{cm} 3$, while the energy density $\rho \approx 5 \times$ $10^{15} \mathrm{~g} / \mathrm{cm}^{3}$. From the equation of state (1) it is obvious that the strange quark matter will always satisfy the energy conditions $\rho \geq P \geq 0$. 
It is generally believed that strange quark matter, consisting of $u, d$ and $s$ quarks, is the most energetically favourable state of baryon matter. Witten [24] suggested that there are two ways of formation of the strange matter: the quarkhadron phase transition in the early universe and the conversion of neutron stars into strange ones at ultrahigh densities. Some theories of strong interactions, e.g. quark bag models, suppose that the breaking of physical vacuum takes place inside hadrons. As a result the vacuum energy densities inside and outside a hadrons become essentially different and the vacuum pressure on a bag wall equilibrates the pressure of quarks thus stabilizing the system. There are several proposed mechanisms for the formation of quark stars. Quark stars are expected to form during the collapse of the core of a massive star after the supernova explosion as a result of a first or second order phase transition, resulting in deconfined quark matter [25]. The protoneutron star core or the neutron star core is a favourable environment for the conversion of ordinary matter to strange quark matter [26]. Another possibility for strange star formation is that some neutron stars in low-mass X-ray binaries can accrete sufficient mass to undergo a phase transition to become strange star [21]. Such a possibility is also supported by an unusual hard X-rayburster [27]. Thismechanism has been proposed as a source of radiation emission for cosmological ray bursts [28]. Other possible astrophysical phenomena related to strange stars are discussed in Ref. [25]. Hence the problem of the collapse of the strange matter is of much interest in the study of strange quark star formation, dynamics and evolution. On the other hand the theoretical understanding of the collapse is of fundamental importance in general relativity.

T. Harko and K.S. Cheng[29] have studied the gravitational collapse of strange matter and analyzed the condition for formation of a naked singularity in the spherically symmetric Vaidya like spacetime. It has been shown that depending on the initial distribution of density and velocity and on the constitutive nature of the collapsing matter, either a black hole or a naked singularity is formed. The purpose of this brief report is to see how the earlier results that were investigated in [29] get modified for non-spherically symmetric (plane symmetric and cylindrically symmetric) spacetime.

The plan of this brief report is as follows: In Sec.II, we obtain the general solution for strange quark matter, with the equation of state $P=\frac{\rho-4 B}{3}$, in non-spherically symmetric spacetime. In Sec.III, we discuss the nature of the singularity by analyzing the equations of the outgoing radial null geodesics. We conclude the report in Sec.IV.

\section{Non-spherical collapse of strange-quark matter}

To expressed the work given in $[29,30,31]$ the metric describing the radial collapse of charge strange quark fluid in toroidal, cylindrical or planar spacetime can be written as

$d s^{2}=-\left[\alpha^{2} r^{2}-\frac{q m(v, r)}{r}\right] d v^{2}+2 d v d r+r^{2}\left(d \theta^{2}+d \varnothing^{2}\right)$

Where $v$ is an advanced Eddington time coordinate, and $\alpha$ is defined by the relation $\alpha=\sqrt{-\Lambda / 3}$. ris the radial coordinate whose range is $0<r<\infty$, and $m(v, r)$ is the mass function giving the gravitational mass inside the sphere of radius $r$. Coordinates $\theta, \varnothing$ describe the two dimensional zero-curvature space generated by the two dimensional commutative Lie group $\mathrm{G}_{2}$ of isometrics[30]. Referring to [30,31], we write the topology of twodimensional space for metric (1) as

Topology of toroidal model is $S \times S$, Cylindrical model has topology $R \times S$ and for planer Symmetrical model has $R \times R$. Ranges for $\theta$ and $\emptyset$ in Toroidal, Cylindrical and Planar models are given by
(i) Toroidal:
$0 \leq \theta<2 \pi$
$0 \leq \emptyset<2 \pi$
(ii) Cylindrical:
$-\infty<\theta<\infty 0 \leq \emptyset<2 \pi$
(iii) Planar:
$-\infty<\theta<\infty-\infty<\emptyset<\infty$

Depending upon the topology of the two-dimensional space, parameter $q$ has different values. In the case of toroidal model, $m(v, r)$ is mass and parameter $q=\frac{2}{\pi}$, for the Cylindrical case $m(v, r)$ is mass per unit length and $q=\frac{4}{\alpha}$ and for Planar symmetrical model $m(v, r)$ is mass per unit area and $q=\frac{2}{\alpha^{2}}$. The values of parameter $q$ are taken from Arnowitt-Deser-Misner (ADM) masses of the corresponding static black hole [31]. The energy momentum tensor for the solution (2) can be written in the form [29, 32].

$T_{\mu v}=T_{\mu v}^{(n)}+T_{\mu v}^{(m)}+E_{\mu v}$,

where

$T_{\mu v}^{(n)}=\sigma(v, r) l_{\mu} l_{v}$

is the component of the matter field that moves along the null hyper surface $v=$ const. , here $\sigma$ is the functions of $v$ and $r$.

The energy momentum tensor of the strange quark matter can be written in the form

$T_{\mu v}^{(m)}=(\rho+p)\left(l_{\mu} \eta_{v}+l_{v} \eta_{\mu}\right)+p g_{\mu v}$

The energy momentum tensor $E_{\mu v}$ of the electromagnetic field is given by

$E_{\mu v}=\frac{1}{4 \pi}\left(F_{\mu \alpha} F_{v}^{\alpha}-\frac{1}{4} g_{\mu v} F_{\alpha \beta} F^{\alpha \beta}\right)$ 
We have consider the two null vectors $l_{\mu}, \eta_{v}$ such that

$l_{\mu}=\delta_{\mu}^{0}, \quad \eta_{\mu}=\frac{1}{2}\left[\alpha^{2} r^{2}-\frac{q m(v, r)}{r}\right] \delta_{\mu}^{0}-\delta_{\mu}^{1}$

$l_{\lambda} l^{\lambda}=\eta_{\lambda} \eta^{\lambda}=0, \quad l_{\lambda} \eta^{\lambda}=-1$

The electromagnetic field tensor $F_{\mu \nu}$ Satisfying Maxwell's equations [19]

$\frac{\partial F_{\mu v}}{\partial x^{\lambda}}+\frac{\partial F_{\lambda \mu}}{\partial x^{v}}+\frac{\partial F_{v \lambda}}{\partial x^{\mu}}=0$

$\frac{1}{\sqrt{-g}} \frac{\partial}{\partial x^{\mu}}\left(\sqrt{-g} F^{\mu v}\right)=-4 \pi J^{\mu}$

Without loss of generality the electromagnetic vector potential can be written as

$A_{\mu}=\frac{e(v)}{r} \delta_{(\mu)}^{(\mu)}$

where $e(v)$ is an arbitrary function of integration. From equations (8) and (9) it follows that only non-vanishing components of $F_{\mu v}$ are

$F_{r v}=-F_{v r}=\frac{e(v)}{r^{2}}$

and hence

$E_{\mu}^{v}=\frac{e^{2}(v)}{r^{4}} \operatorname{diag}(-1,1,-1,1)$

From the Einstein's field equation for the energy momentum tensor (3) can be written as

$\sigma=\frac{q \dot{m}}{8 \pi r^{2}}$

$\rho=\frac{q m^{\prime}-3 \alpha^{2} r^{2}}{8 \pi r^{2}}-\frac{q^{2} e^{2}(v)}{8 \pi r^{4}}$

$P=\frac{6 \alpha^{2} r-q m^{\prime \prime}}{16 \pi r}-\frac{q^{2} e^{2}(v)}{16 \pi r^{4}}$

where an over-dot and dash stand for differentiation with respect to $v$ and $r$ respectively.

The energy conditions for the above type of fluids are given by [29, 32].

i) The weak and strong energy conditions:

$\sigma>0, \quad \rho \geq 0, \quad P \geq 0$

ii) The dominant energy conditions:

$\sigma>0, \quad \rho \geq P \geq 0$

Substituting the values of Eqs. (14) and (15) in the bag equation of state (1), we obtain the following differential equation

$3 q m^{\prime \prime} r^{2}+2 q m^{\prime} r=64 \pi B r^{3}+24 \alpha^{2} r^{3}-\frac{q^{2} e^{2}(v)}{r}$

solving this Cauchy's homogeneous differential equation we obtain the following general solution

$q m(v, r)=q g(v)+q h(v) r^{1 / 3}+A r^{3}-\frac{q^{2} e^{2}(v)}{4 r}$

The two arbitrary functions $g(v)$ and $h(v)$ would be restricted only by the energy conditions and $A=\left(\frac{8 \pi B}{3}\right)+$ $\alpha^{2}$ substituting the mass function $m(v, r)$ from equation (19) into the equation (2), we obtain the following solution to the Einstein equations for a collapsing null strange quark matter in toroidal (cylindrical or planar) spacetime.

$d s^{2}=-\left[\alpha^{2} r^{2}-\frac{q g(v)}{r}-\frac{q h(v)}{r^{2 / 3}}-A r^{2}+\frac{q^{2} e^{2}(v)}{4 r^{2}}\right] d v^{2}+2 d v d r+r^{2}\left(d \theta^{2}+d \emptyset^{2}\right)$

The magnetic monopole solution can be characterized by its mass and radius, some spontaneously broken gauge theories contain magnetic monopoles which have the remarkable property that, despite being particles in a quantum theory, they are described by a classical field configuration. This is because in the limit of weak gauge coupling e their Compton wavelength $\sim e / v$ is much less than the radius $\sim 1 /(e v)$ of the classical monopole solution.

Now, we coupling the above mass function of strange quark matter with the monopole field thus

$q m(v, r)=a r+q g(v)+q h(v) r^{1 / 3}+A r^{3}-\frac{q^{2} e^{2}(v)}{4 r}$

called the monopole strange quark matter.

substituting the mass function $m(v, r)$ from equation (21) into equation (2), we obtain the solution to the Einstein equations for collapsing monopole strange quark matter in toroidal (cylindrical or planar) spacetime.

$d s^{2}=-\left[\alpha^{2} r^{2}-a-\frac{q g(v)}{r}-\frac{q h(v)}{r^{2 / 3}}-A r^{2}+\frac{q^{2} e^{2}(v)}{4 r^{2}}\right] d v^{2}+2 d v d r+r^{2}\left(d \theta^{2}+d \emptyset^{2}\right)$

Inserting the mass function (21) into the equation (13) - (15) we obtain the following quantities

$\sigma=\frac{1}{8 \pi r^{2}}\left[q \dot{g}(v)+q \dot{h}(v) r^{1 / 3}-\frac{q^{2} e(v) \dot{e}(v)}{2 r}\right]$

$\rho=\frac{1}{8 \pi r^{2}}\left[a+\frac{1}{3} q h(v) r^{-2 / 3}+8 \pi B r^{2}-\frac{3}{4 r^{2}} q^{2} e^{2}(v)\right]$

$P=\frac{1}{16 \pi r}\left[\frac{2}{9} q h(v) r^{-5 / 3}-16 \pi B r-\frac{q^{2} e^{2}(v)}{2 r^{3}}\right]$ 
we note that with proper choice of $g(v)$ and $h(v)$, weak and strong energy conditions are satisfied. Due to the bag equation of state (1) always satisfies the condition $\rho \geq P \geq 0$ and thus dominant energy condition holds too.

\section{Nature of the Singularity}

Following the method of Dwivedi and Joshi [8] we know examine under what conditions on $m(v, r)$ such collapse leads to a naked singularity and whether this singularity is a strong curvature one or not. The existence of naked singularity or otherwise can be determined by examining the behaviour of radial null geodesic. If they terminate at the singularity in the past with a definite positive tangent vector, then the singularity is naked. If the tangent vector is not positive in the limit as one approaches the singularity, then the singularity is covered[33].

To analyze the structure of the collapse we need to consider the radial null geodesic defined by $d s^{2}=0$ taking into the account $\dot{\theta}=\dot{\emptyset}=0$ then the equation (22) becomes

$\frac{d r}{d v}=\frac{1}{2}\left[\alpha^{2} r^{2}-a-\frac{q g(v)}{r}-\frac{q h(v)}{r^{2 / 3}}-A r^{2}+\frac{q^{2} e^{2}(v)}{4 r^{2}}\right]$

In general, the above equation does not yield analytic solution. However if $q g(v) \propto v, q h(v) \propto v^{2 / 3}$

and $q^{2} e^{2}(v) \propto v^{2}$, then this equation becomes homogeneous and can be solved in terms of elementary functions.

In particular, let us choosing

$q g(v)=h v, \quad q h(v)=\beta v^{2 / 3}$ and $q^{2} e^{2}(v)=\delta^{2} v^{2}$

for some values of $\lambda>0, \quad \beta>0$ and $\delta \geq 0$ then the equation (26) becomes

$\frac{d u}{d r}=\frac{2}{\left[\alpha^{2} r^{2}-a-\frac{\lambda v}{r}-\frac{\beta v^{2 / 3}}{r^{2 / 3}}-A r^{2}+\frac{\delta^{2} v^{2}}{4 r^{2}}\right]}$

It can be observed from equation (28) that this equation has singularity at $r \rightarrow 0, v \rightarrow 0$. To discuss the nature of this singularity the radial and non-radial outgoing non-space like geodesics terminating at this singularity in the past, we need to consider the limiting value of $X=\frac{v}{r}$ along a singular geodesic at the singularity is approached[34, 35, 36].

Let,

$X_{0}=\lim _{\substack{v \rightarrow 0 \\ r \rightarrow 0}} \frac{d v}{d r}$

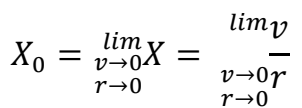

Hence from equation (28), we have

$X_{0}=\frac{8}{-4 a-4 \lambda X_{0}-4 \beta X_{0}^{2 / 3}+\delta^{2} X_{0}^{2}}$

i.e. $\delta^{2} X_{0}^{3}-4 \lambda X_{0}^{2}-4 \beta X_{0}^{5 / 3}-4 a X_{0}-8=0$

This algebraic equation which would ultimately decide the end state of collapse: a black hole or a naked singularity. Thus by analyzing this algebraic equation, the nature of singularity can be determined. The central shell focusing singularity would at least be locally naked,if equation (31) admits one or more positive real roots [see the Ref.35]. The values of the roots give the tangents of the escaping geodesics near the singularity. When there are no positive real roots to equation (31), there are no outgoing future directed null geodesics emanating from the singularity. Thus the occurrence of positive roots would imply the violation of the strong $\mathrm{CCH}$, though not necessary of the weak form. Hence in the absence of positive real roots, the collapse will always lead to a black hole. The positive root would define the range for the tangent slopes from which the null geodesics can escape to infinity.

Setting $X_{0}=p^{3}$ in equation (31), we obtain

$\delta^{2} p^{9}-4 \lambda p^{6}-4 \beta p^{5}-4 a p^{3}-8=0$

To investigate the nature of the root of equation (32), the following rule from the theory of equations may be useful: Every equation of odd degree has at least one real root whose sign is opposite to that of its last term, the coefficient of the first term being positive. According to this fact, equation (32) has at least one positive root.

In particular $\delta^{2}=0.01, \lambda=0.1, \quad \beta=0.1, \quad a=0.1$ then one of root of equation (32) is

$p=3.7277$. After back substitution gives $X_{0}=51.7992$ this positive root of equation (31), indicates that the singularity is naked.

Now we consider some different values of $\delta^{2}, \lambda, \beta$, and $a$ to study the nature of singularity of equation (32) and back substitution to gives the root of equation (31).

For fixed $\delta^{2}=0.01$ and $\beta=0.1$ then the equation (32) reduces to the form

$0.01 p^{9}-4 \lambda p^{6}-4(0.1) p^{5}-4 a p^{3}-8=0$ 
Effect of Monopole Field on the Non-spherical Gravitational Collapse of Strange-quark Matter.

then the roots of equation (33) obtained for different values of $\lambda$ and $a$ and after back substitution gives the values of $X_{0}$ in non-spherical gravitational collapse of monopole strange quark matter.

Table 1: Values of $X_{0}$ for different values of $\lambda$ and $a$.

\begin{tabular}{|l|l|l|l|l|l|}
\hline \multirow{2}{*}{$\lambda$} & \multicolumn{5}{|c|}{$X_{0}$} \\
\cline { 2 - 6 }$\lambda$ & $\mathrm{a}=0.1$ & $\mathrm{a}=0.25$ & $\mathrm{a}=0.5$ & $\mathrm{a}=0.75$ & $\mathrm{a}=0.85$ \\
\hline 0.1 & 51.7992 & 52.8399 & 54.4917 & 56.0588 & 56.6629 \\
\hline 0.2 & 89.4886 & 90.1323 & 91.1797 & 92.2045 & 92.6031 \\
\hline 0.3 & 128.291 & 128.7494 & 129.5005 & 130.239 & 130.5399 \\
\hline 0.4 & 167.5211 & 167.8769 & 168.4618 & 169.039 & 169.2684 \\
\hline 0.5 & 206.9704 & 207.2645 & 207.7376 & 208.2115 & 208.4013 \\
\hline 0.6 & 246.5509 & 246.7987 & 247.2002 & 247.5903 & 247.756 \\
\hline 0.7 & 286.2172 & 286.4258 & 286.7779 & 287.1172 & 287.2608 \\
\hline 0.8 & 325.9448 & 326.1295 & 326.4281 & 326.7269 & 326.855 \\
\hline 0.9 & 365.7099 & 365.8787 & 366.1397 & 366.4161 & 366.5083 \\
\hline
\end{tabular}

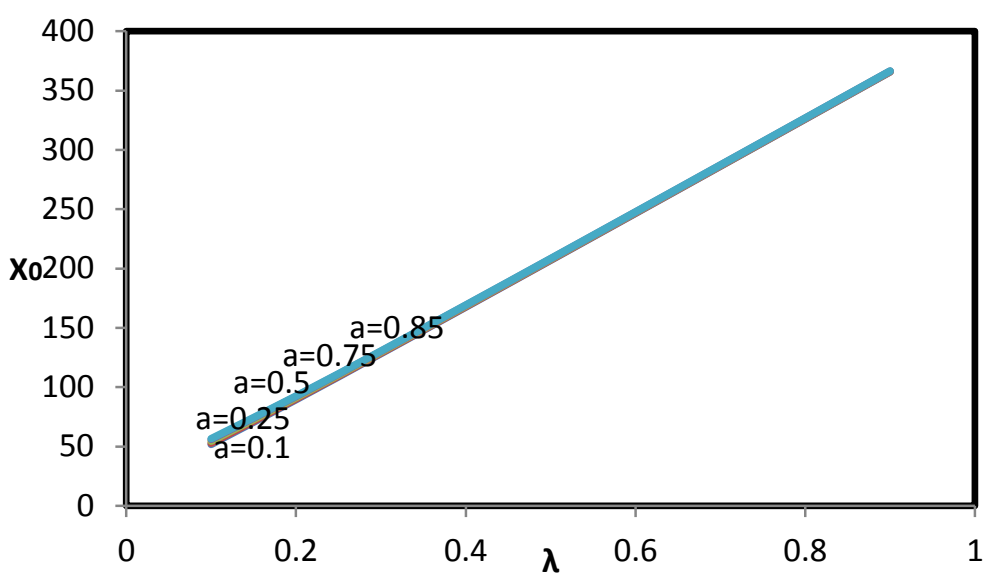

Fig. 1: Graph of the values of $X_{0}$ against the values of $\lambda$.

The nature of singularity is illustrated through the above graphs. We note that the values of $X_{0}$ increases for increasing the value of $\lambda$, it means the lower value shifted towards the higher value.

For fixed $a=0.25$ and $\beta=0.1$ then the equation (32) reduces to the form

$\delta^{2} p^{9}-4 \lambda p^{6}-4(0.1) p^{5}-4(0.25) p^{3}-8=0$

then the roots of equation (34) obtained for different values of $\lambda$ and $a$ and after back substitution gives the values of $X_{0}$ in non-spherical gravitational collapse of monopole strange quark matter.

Table 2: Values of $X_{0}$ for different values of $\lambda$ and $\delta^{2}$.

\begin{tabular}{|l|l|l|l|l|l|}
\hline \multirow{2}{*}{$\delta^{2}$} & \multicolumn{5}{|c|}{$X_{0}$} \\
\cline { 2 - 6 } & $\lambda=0.1$ & $\lambda=0.3$ & $\lambda=0.5$ & $\lambda=0.7$ & $\lambda=0.9$ \\
\hline 0.01 & 52.8399 & 128.7494 & 207.2645 & 286.4258 & 365.8787 \\
\hline 0.02 & 28.751 & 65.8071 & 104.7535 & 144.1828 & 183.8027 \\
\hline 0.03 & 20.4719 & 44.6406 & 70.4245 & 96.6141 & 122.9711 \\
\hline 0.04 & 16.2443 & 33.9965 & 53.1998 & 72.7781 & 92.5049 \\
\hline 0.05 & 13.6656 & 27.5819 & 42.8419 & 58.4517 & 74.2045 \\
\hline 0.06 & 11.9211 & 23.2956 & 35.9207 & 48.8874 & 61.9863 \\
\hline 0.07 & 10.6582 & 20.2259 & 30.971 & 42.0462 & 53.255 \\
\hline 0.08 & 9.6977 & 17.9189 & 27.2546 & 36.9127 & 46.7027 \\
\hline 0.09 & 8.9406 & 16.1233 & 24.3613 & 32.9188 & 41.6016 \\
\hline
\end{tabular}




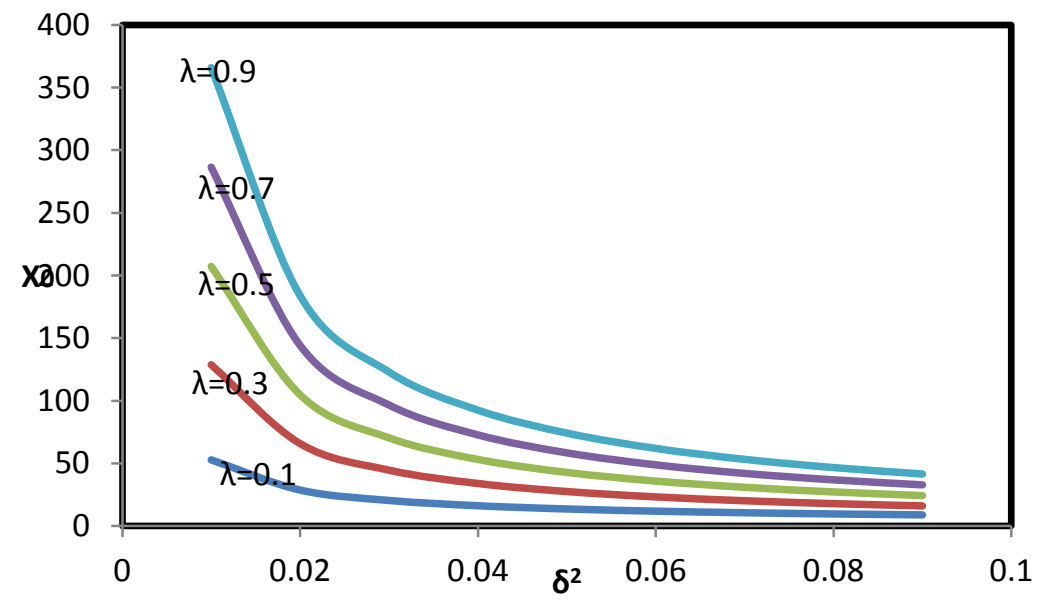

Fig. 2: Graph of the values of $X_{0}$ against the values of $\delta^{2}$.

The graph shows that initially the values is at peak, when we increase the values of electric charge the values of $X_{0}$ decreases very rapidly, it means the peak shifted towards lower values.

For fixed $\lambda=0.5$ and $\beta=0.1$ then the equation (32) reduces to the form $\delta^{2} p^{9}-4(0.5) p^{6}-4(0.1) p^{5}-4 a p^{3}-8=0$

then the roots of equation (35) obtained for different values of $\lambda$ and $a$ and after back substitution gives the values of $X_{0}$ in non-spherical gravitational collapse of monopole strange quark matter.

Table 3: Values of $X_{0}$ for different values of $a$ and $\delta^{2}$.

\begin{tabular}{|l|l|l|l|l|l|}
\hline \multirow{2}{*}{$\delta^{2}$} & \multicolumn{5}{|c|}{$X_{0}$} \\
\cline { 2 - 6 } & $\mathrm{a}=0.1$ & $\mathrm{a}=0.25$ & $\mathrm{a}=0.5$ & $\mathrm{a}=0.75$ & $\mathrm{a}=0.85$ \\
\hline 0.01 & 206.9704 & 207.2645 & 207.7376 & 208.2115 & 208.4013 \\
\hline 0.02 & 104.4738 & 104.7535 & 105.2209 & 105.6829 & 105.8708 \\
\hline 0.03 & 70.1435 & 70.4245 & 70.8808 & 71.334 & 71.5147 \\
\hline 0.04 & 52.9244 & 53.1998 & 53.6509 & 54.1002 & 54.2764 \\
\hline 0.05 & 42.567 & 42.8419 & 43.2879 & 43.7258 & 43.9011 \\
\hline 0.06 & 35.6503 & 35.9207 & 36.3634 & 36.7964 & 36.966 \\
\hline 0.07 & 30.7025 & 30.971 & 31.4079 & 31.8339 & 31.9999 \\
\hline 0.08 & 26.9892 & 27.2546 & 27.6861 & 28.1056 & 28.2695 \\
\hline 0.09 & 24.0975 & 24.3613 & 24.7873 & 25.2001 & 25.3629 \\
\hline
\end{tabular}

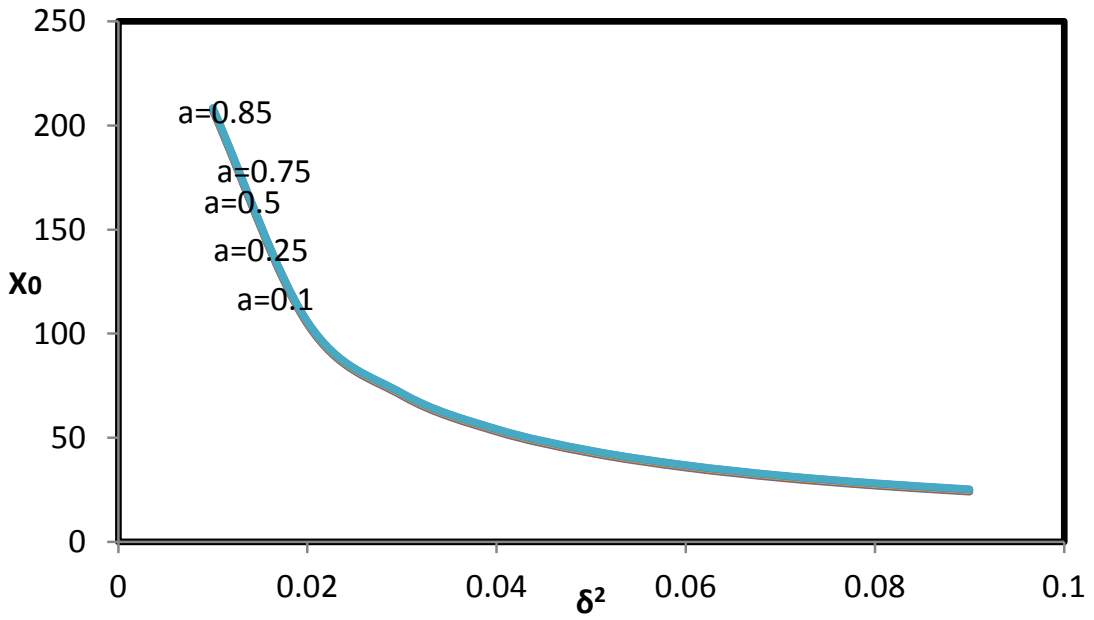

Fig.3: Graph of the values of $X_{0}$ against the values of $\delta^{2}$.

From the graph it is shown that when increase the value of $\lambda$ the values of $X_{0}$ increases very rapidly and also decreases rapidly for increasing charge.

For fixed $\lambda=0.1$ and $a=0.5$ then the equation (32) reduces to the form 
$\delta^{2} p^{9}-4(0.1) p^{6}-4 \beta p^{5}-4(0.5) p^{3}-8=0$

then the roots of equation (36) obtained for different values of $\lambda$ and $a$ and after back substitution gives the values of $X_{0}$ in non-spherical gravitational collapse of monopole strange quark matter.

Table 4: Values of $X_{0}$ for different values of $\beta$ and $\delta^{2}$.

\begin{tabular}{|l|l|l|l|l|l|}
\hline \multirow{2}{*}{$\beta$} & \multicolumn{5}{|c|}{$X_{0}$} \\
\cline { 2 - 6 } & $\delta 2=0.01$ & $\delta 2=0.03$ & $\delta 2=0.05$ & $\delta 2=0.07$ & $\delta 2=0.09$ \\
\hline 0.1 & 54.4917 & 21.741 & 14.7205 & 11.5662 & 9.7428 \\
\hline 0.2 & 63.4162 & 25.4355 & 17.0977 & 13.3239 & 11.1374 \\
\hline 0.3 & 71.8097 & 28.974 & 19.4152 & 15.0582 & 12.526 \\
\hline 0.4 & 79.7958 & 32.3794 & 21.6663 & 16.7576 & 13.8984 \\
\hline 0.5 & 87.4515 & 35.6665 & 23.8531 & 18.4207 & 15.2456 \\
\hline 0.6 & 94.8251 & 38.848 & 25.9817 & 20.0461 & 16.5697 \\
\hline 0.7 & 101.9719 & 41.9374 & 28.0584 & 21.6337 & 17.8676 \\
\hline 0.8 & 108.914 & 44.9471 & 30.0831 & 23.1906 & 19.1392 \\
\hline 0.9 & 115.6721 & 47.8875 & 32.0665 & 24.7134 & 20.3889 \\
\hline
\end{tabular}

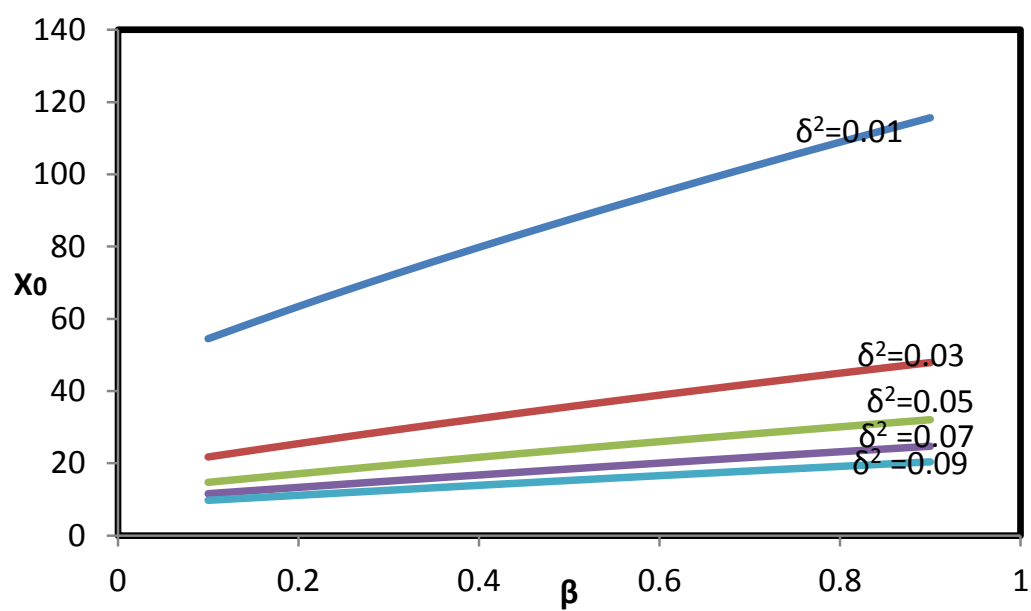

Fig. 4: Graph of the values of $X_{0}$ against the values of $\delta^{2}$.

From the graph it is observed that increasing the value of $\beta$ the values of $X_{0}$ increases and also it is note that when increases the electric charge the values of $X_{0}$ decreases.

In the above discussion we have considered the non-spherical gravitational collapse of monopole charged strange quark matter. It would be interesting to see whether the gravitational collapse of uncharged (i.e. $q^{2} e^{2}(u)=0$ ) monopole strange quark matter leads to a naked singularity or black hole.

For the uncharged case, put $q^{2} e^{2}(u)=0$ into equation (21), then equation (31) becomes $4 \lambda X_{0}^{2}+4 \beta X_{0}^{5 / 3}+4 a X_{0}+8=0$

It is seen that the coefficients in equation (37) are positive, it can be argued by theory of equations that equation (37) cannot have positive roots. In other word we say that outgoing radial null geodesics having definite tangent at the singularity in the past are absent, hence here is no naked singularity. Thus collapse proceeds to form toroidal, cylindrical or planar black holes.

\section{Conclusion}

In the present paper we consider the collapse of a non-spherical charge monopole strange quark matter. The possible occurrence of a central naked singularity has also been investigated and it has been shown that singularity is to be visible by the outside observer, i.e. a naked singularity is formed. The spacetime singularity to be locally naked is that the algebraic equation (31) should have at least one or more positive root. Hence existence of the positive roots of equation (31) is a counter example to the strong version of the $\mathrm{CCH}$. In the absence of the proof of any version of $\mathrm{CCH}$, such examples remain the only tool to study this important and unresolved problem.

The interesting feature which emerges that charge plays a crucial role in the non-spherical gravitational collapse of monopole strange quark matter and it leads to a naked singularity. Whereas gravitational collapse of neutral monopole strange quark matter proceeds to form a black hole. Thus the non-spherical gravitational collapse of charged monopole strange quark matter contradicts the $\mathrm{CCH}$, whereas collapse of neutral monopole strange quark matter respects it. 
In the paper K. D. Patil, S. S. Zade and A. N. Mohod shown that charge plays a major role in the formation of naked singularity whereas the charge is absent then it produces a black hole. In addition to this monopole spacetime does not affect the nature of singularity hence monopole spacetime does not play any fundamental role in the formation of naked singularity.

\section{References}

[1] D M Eardley and L Smar, Time function in numerical relativity: Marginally bound dust collapse, Phys. Rev,D 19, 1979, 22392259.

[2] D Christodoulou, Violation of cosmic censorship in gravitational collapse of a dust cloud, Commun. Math. Phys., 93, 1984, 171195.

[3] R P A C Newman, Strength of naked singularities in Tolman-Bondi space-times, Class. Quantum Grav.,3, $1986,527$.

[4] K D Patil and U S Thool, Spherical and non-spherical gravitational collapse in Husain space-time,Int. J. Mod. Phys., D 14(6), $2005,873-882$

[5] K D Patil, Structure of radial null geodesics in higher-dimensional dust collapse, Phys. Rev. D 67, $2003,024017$.

[6] A Papapetrou, in A Random Walk in Relativity and Gravitation, eds. N Dadhich, J K Rao, J V Narlikar and C V Vishveshwara (Wiley, New York, 1985)

[7] B Waugh and K Lake, Double null coordinates for the Vaidya metric, Phys. Rev. D34, 1986, 2978-2984.

[8] I H Dwivedi and P S Joshi,On the nature of naked singularities in Vaidya spacetime,Class. Quantum. Grav.6,1989, 1599.

[9] K D Patil and U S Thool, Spherical and non-spherical gravitational collapse in monopole anti- de-sitter vaidya space time, Int. J. Mod. Phys. D 15(11), 2006, 1977-1984.

[10] A Ori and T Piran, Naked singularities in self-similar spherical gravitational collapse,Phys. Rev. Lett.59, 1987, 2137-2140.

[11] R. Penrose, Gravitational collapse: The role of general relativity, Riv. Nuovo Cimento 1, 1969, 252.

[12] P. S. Joshi and R. Goswami, Role of initial data in spherical collapse,Physical Review D 69, $2004,064027$.

[13] R. Goswami and P. S. Joshi, What role pressures play to determine the final end-state of gravitational collapse?Class. Quantum Grav. 19, 2002, 5229-5234.

[14] C. J. S. Clarke, Class. Quantum Grav.10, 1375 (1994); R. M. Wald, gr-qc/9710068; S. Jhingan and G. Magli, gr-qc/9902041; T. P. Singh, J. Astrophys. and Astron. 20, 221 (1999).

[15] P. S. Joshi and I. H. Dwivedi, Commun. Math. Phys., 146, 333, 1992; Lett. Math Phys. 27, 235, 1993; Commun. Math. Phys.166 117-128 (1994); Class. Quantum Grav.16, 1999, 41-59.

[16] B. Waugh and K. Lake, Self focusing singularities in spherically symmetric self-similar spacetimes, Phys. Rev. D 40, 1989, 2137; Phys. Rev. D 42, 1990, 1068.

[17] M. E. Cahill and A. H. Taub, Commun. Math. Phys.21, 1971, 1.

[18] J. F. V. Rocha, A. Wang and N. O. Santos, Gravitational collapse of perfect fluid,Phys. Lett. A 255, 1999, 213-220

[19] Edward Farhi and Angela Olinto, Strange stars, The Astrophysical Journal,310, 1986, 261-272.

[20] Somenath Chakraborty, Phys. Rev. D43, 1991, 627-630.

[21] K S Cheng and Z G Dai, Conversion Of Neutron Stars To Strange Stars As An Origin Of Gamma-Ray Bursts,Phys. Rev. Lett.77 No.7, 1996, 1210-1213.

[22] Anbo Chen, Tianhong YuAnd Renxin Xu, The birth of quark stars: Photon-driven supernovae, Astro-ph/0605285, 2006

[23] Charles Alcock and Edward Farhi, Phys. Rev. D32, No.6, 1985, 1273-1279.

[24] E. Witten, Cosmic separation of phases, Phys. Rev. D 30, 1984, 272-285.

[25] K. S. Cheng, Z. G. Dai, T. Lu, Steller astrophysics, Int. J. Mod. Phys. D 7, 1998, 139.

[26] Z. G. Dai, Q. H. Peng, T. Lu, Astrophys. J.440, 1995, 815.

[27] K. S. Cheng, Z. G. Dai, D. M. Wei, T. Lu, Is GROJ1744-28 A Strange Stars?Science 280, 1998, 407-409.

[28] K. S. Cheng, Z. G. Dai, Are Soft $\gamma$-Ray Repeaters Strange Stars? Phys. Rev. Lett. 80, 1998, 18-21.

[29] T Harko and K S Cheng, Collapsing strange quark matter in Vaidya geometry, Phys. Lett. A266, 2000, 249

[30] J P S Lemos,Collapsing shells of radiation in anti-de Sitter spacetimes and the hoop and cosmic censorship conjectures, Phys. Rev. D59, 044020, 1999.

[31] J P S Lemos, Gravitational collapse to toroidal, cylindrical, and planar black holes, Phys. Rev. D57, 1998, 4600-4605.

[32] Anzong Wang and Yumei Wu, Generalized Vaidya solution, Gen, Relativ. Grav.31, 1999 (1).

[33] K D Patil,Gravitational collapse in higher dimensional charged-Vaidya spacetimePramana J. Phys., Vol. 60,2003,423-431.(3)

[34] P S Joshi, Global Aspects in gravitation and cosmology, (Clarendon, Oxford, 1993).

[35] S G Ghosh and N Dadhich, Gravitational collapse of null strange quark fluid and cosmic censorship, arXiv: gr-qc/0211019, V 1, 2002.

[36] S G Ghosh and N Dadhich, On naked singularities in higher dimensional Vaidyaspacetimes, arXiv: gr-qc/0105085 V 2, 2001. 